\title{
A Shared Task on Bandit Learning for Machine Translation
}

\author{
Artem Sokolov $^{*, \diamond}$ and Julia Kreutzer ${ }^{\diamond}$ and Kellen Sunderland ${ }^{*}$ and Pavel Danchenko* \\ and Witold Szymaniak* and Hagen Fürstenau* and Stefan Riezler ${ }^{\diamond}$ \\ ${ }^{*}$ Amazon Development Center Germany, Berlin and ${ }^{\diamond}$ Heidelberg University, Germany
}

\begin{abstract}
We introduce and describe the results of a novel shared task on bandit learning for machine translation. The task was organized jointly by Amazon and Heidelberg University for the first time at the Second Conference on Machine Translation (WMT 2017). The goal of the task is to encourage research on learning machine translation from weak user feedback instead of human references or post-edits. On each of a sequence of rounds, a machine translation system is required to propose a translation for an input, and receives a real-valued estimate of the quality of the proposed translation for learning. This paper describes the shared task's learning and evaluation setup, using services hosted on Amazon Web Services (AWS), the data and evaluation metrics, and the results of various machine translation architectures and learning protocols.
\end{abstract}

\section{Introduction}

Bandit Learning for machine translation (MT) is a framework to train and improve MT systems by learning from weak or partial feedback: Instead of a gold-standard human-generated translation, the learner only receives feedback to a single proposed translation (hence the term 'partial'), in form of a translation quality judgement (a real number which can be as weak as a binary acceptance/rejection decision).

In the shared task, user feedback was simulated by a service hosted on Amazon Web Services (AWS). Participants can submit translations and receive feedback on translation quality. This is used to adapt an out-of-domain MT model, pre-trained on mostly news texts, to a new do- main (e-commerce), for the translation direction of German (DE) to English (EN). While in our setup feedback was simulated by evaluating a reward function on the predicted translation against a gold standard reference, the reference translation itself was never revealed to the learner, neither at training nor at test time. This learning scenario has been investigated under the names of learning from bandit feedback ${ }^{1}$ or reinforcement learning $(\mathrm{RL})^{2}$, and has important real world applications such as online advertising (Chapelle et al., 2014). In the advertising application, the problem is to select the best advertisement for a user visiting a publisher page. A key element is to estimate the click-through rate (CTR), i.e., the probability that an ad will be clicked by a user so that the advertiser has to pay. This probability is modeled by features representing user, page, and ad, and is estimated by trading off exploration (a new ad needs to be displayed in order to learn its click-through rate) and exploitation (displaying the ad with the current best estimate is better in the short term) in displaying ads to users.

In analogy to the online advertising scenario, one could imagine a scenario of personalization in machine translation where translations have to be adapted to the user's specific purpose and domain. Similar to online advertising, where it is unrealistic to expect more detailed feedback than a user click on a displayed ad, the feedback in adaptive machine translation should be weaker than a reference translation or a post-edit created by

\footnotetext{
${ }^{1}$ The name is inherited from a model where in each round a gambler pulls an arm of a different slot machine ('onearmed bandit'), with the goal of maximizing his reward relative to the maximal possible reward, without apriori knowledge of the optimal slot machine. See Bubeck and CesaBianchi (2012) for an overview.

${ }^{2}$ See Sutton and Barto (1998) and Szepesvári (2009) for an overview of algorithms for reinforcement learning and their relation to bandit learning.
} 
a professional translator. Instead, the goal is to elicit binary or real-valued judgments of translation quality from laymen users (for example, Graham et al. (2016) show that consistent assessments of real-valued translation quality can be provided by crowdsourcing), or to infer feedback signals from user interactions with the translated content on a web page (for example, by interpreting a copy-paste action of the MT output as positive quality signal, and a correction as a negative quality signal). The goal of this shared task is to evaluate existing algorithms for learning MT systems from weak feedback (Sokolov et al., 2015, 2016a; Kreutzer et al., 2017) on real-world data and compare them to new algorithms, with a focus on performing online learning efficiently and effectively from bandit feedback, i.e. the best algorithms are those that perform fast online learning and, simultaneously, achieve high translation quality.

In the following, we present a description of the protocol and infrastructure of our online learning task, and of the data for pretraining, online training, and evaluation (Section 2). We introduce the online and batch evaluation metrics used in the shared task (Section 3), and describe static baseline systems (Section 4) and submitted online learning systems (Section 5). We present and discuss the results of the task (Section 6-7), showing that NMT systems with batch domain adaptation provide very good baselines, however, online learning based on SMT or NMT can catch up over time by adapting to the provided feedback.

\section{Task Description}

Our shared task setup follows an online learning protocol, where on each iteration, the learner receives a source sentence, proposes a translation, and is rewarded in form of a task sentence-level metric evaluation of the proposed translation with respect to a hidden reference. The learner does not know what the correct translation (reference) looks like, nor what would have happened if it had proposed a different translation. Thus, we implemented two constraints to guarantee this scenario of online learning from weak feedback. First, sentences had to be translated one by one, i.e. the next source sentence could only be received after the translation to the previous sentence was sent off. Second, feedback could be obtained only for a single translation of any given source sentence.

In our shared task, the participant systems inter-

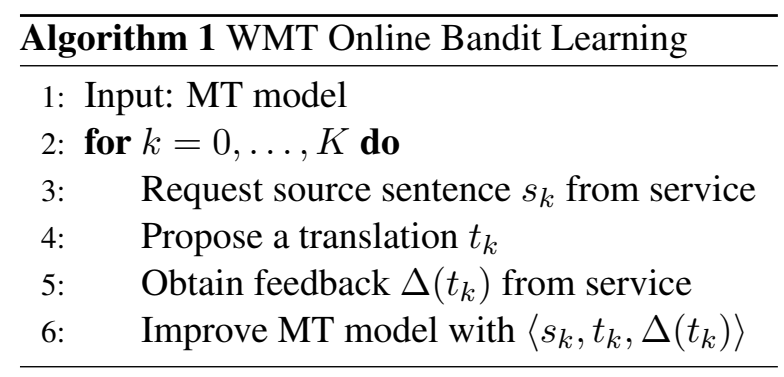

act online with an AWS-hosted service as shown in Algorithm 1. The service provides a source sentence to the learner (line 3), and provides feedback (line 5) to the translation predicted by the learner (line 4). The learner updates its parameters using the feedback (line 6) and continues to the next example. We did not impose any restriction on how the learner could use the feedback to improve future translations.

Infrastructure. We provided three AWS-hosted environments, that correspond to the three phases of the shared task:

1. Mock service, to test the client API (optional): hosted a tiny in-domain dataset (48 sentences).

2. Development service to tune algorithms and hyperparameters (optional): ran on a larger in-domain dataset $(40,000$ sentences). Several passes were allowed and two evaluation metrics were communicated to the participants via the leaderboard.

3. Training service (mandatory): served sources from a large in-domain dataset $(1,297,974$ sentences). Participants had to consume a fixed number of samples during the allocated online learning period to be eligible for final evaluation.

We built the shared task around the following AWS services:

- API Gateway (authentication, rate limiting, client API SDK);

- Lambda (computation);

- DynamoDB (data storage);

- CloudWatch (logging and monitoring).

In more detail, service endpoints were implemented using API Gateway, that gave us access, on a participant level, to throttle requests rates, manage accounts, etc. API Gateway enabled easy management of our public-facing endpoints and 


\begin{tabular}{|c|c|c|c|}
\hline source & reference $(\mathrm{PE})$ & PE direction & PE modification \\
\hline $\begin{array}{l}\text { schwarz gr.xxl / xxxl } \\
147 \mathrm{~cm} \\
\text { für starke, glänzende nägel } \\
\text { seemless verarbeitung } \\
\text { brenndauer : mindestens } 40 \text { stunden } \\
\text { maschinenwaschbar bei } 30^{\circ} \mathrm{c} \\
32 \text { unzen volumen } \\
\text { material : } 1050 \text { denier nylon } \\
\text { für e-gitarre entworfen }\end{array}$ & $\begin{array}{l}\text { black, size xxl / xxxl } \\
147 \mathrm{~cm} \\
\text { great for strengthen your nails and enhance shine } \\
\text { seamless processing } \\
40 \text { hour minimum burn time } \\
\text { machine washable at } 30 \text { degrees. } \\
\text { 32-ounce capacity } \\
\text { material : } 1050 \mathrm{~d} \text { nylon. } \\
\text { designed for electric guitar }\end{array}$ & $\begin{array}{l}\text { DE-EN } \\
\text { DE-EN } \\
\text { EN-DE } \\
\text { DE-EN } \\
\text { DE-EN } \\
\text { DE-EN } \\
\text { DE-EN } \\
\text { EN-DE } \\
\text { DE-EN }\end{array}$ & $\begin{array}{c}\text { fixed errors in source, expanded abbreviation } \\
\text { fixed errors in source } \\
\text { poor quality source (EN) used as reference } \\
\text { source typo corrected in reference } \\
\text { translation rewritten for readability } \\
\text { literal expansion of the degree symbol } \\
\text { language-specific typography } \\
\text { expanded source (EN) abbreviation used as reference } \\
\text { abbreviation expanded }\end{array}$ \\
\hline
\end{tabular}

Table 1: Examples for non-literal PEs in the e-commerce data: The first two columns show examples ${ }^{3}$ of source sentences and PEs used as reference translations in the shared task. The last two columns show the direction of translation post-editing, and a description of the modifications applied by the editors.

environments, and provided integrated metrics and notifications, which we monitored closely during the shared task. Data storage was implemented using DynamoDB - a NoSQL storage database which allows dynamic scaling of our back-end to match the varied requirements of the different shared task phases. The state management (e.g., forbidding multiple requests), source sentence serving, feedback calculation, keeping track of participant's progress and result processing was implemented using Lambda - a serverless compute architecture that dispenses with setting up and monitoring a dedicated server infrastructure. CloudWatch service was used to analyze logs in order to trace down errors, general monitoring and sending alarms to the shared task API maintainers. In addition to the service development, we also developed a small SDK consisting of code samples and helper libraries in Python and Java to help participants in developing their clients, as well as a leaderboard that showed the results during the development phase.

Data. For training initial or seed MT systems (the input to Algorithm 1), out-of-domain parallel data was restricted to DE-EN parts of Europarl v7, NewsCommentary v12, CommonCrawl and Rapid data from the WMT 2017 News Translation (constrained) task ${ }^{4}$. Furthermore, monolingual EN data from the constrained task was allowed. Tuning of the out-of-domain systems had to be done on the newstest2016-deen set.

The in-domain parallel data for online learning was taken from the e-commerce domain: The corpus was provided by Amazon and had been sampled from a large real-world collection of postedited (PE'ed) translations of actual product descriptions. Since post-editors were following cer-

\footnotetext{
${ }^{3}$ Examples selected by Khanh Ngyuen.

${ }^{4}$ statmt.org/wmt 17/translation-task.html
}

tain rules aimed at improving customer experience on the Amazon retail website (improving readability, correction of typos, rewriting of uncommon abbreviations, removing irrelevant information, etc.), naturally the resulting PEs were not always literal, sometimes adding or deleting a considerable number of tokens and resulting in low feedback BLEU scores for submitted literal translations (see Table 1 for examples). Consequently, the participants had to solve two difficult problems - domain adaptation and learning from bandit feedback. In addition, to simulate the level of noise normally encountered in real-world MT applications, and to test noise-robustness of the bandit learning algorithms, approximately half of the parallel in-domain data was sourced from the ENDE post-editing direction and reversed.

All data was preprocessed with Moses scripts (removing non-printing characters, replacing and normalizing unicode punctuation, lowercasing, pretokenizing and tokenizing). No DE-side compound splitting was used, permitting custom participant decisions. Since the learning data came from a substantially different domain than the outof-domain parallel texts, it had a large number of out-of-vocabulary (OOV) terms, aggravated by the high frequency of long product numbers and unique vendor names. To reduce the OOV rate we additionally filtered out all parallel sentences where the source contained more than one numeral (with a whitespace in between) and normalized floating point delimiters in both languages to a period. The resulting average OOV token rate with respect to the out-of-domain parallel training data (assuming the above preprocessing) is $\simeq 2 \%$ for $\mathrm{EN}$ and $\simeq 6 \%$ for DE data side. Statistics on the length distribution of in-domain and out-ofdomain data is given in Table 2 .

For all services, the sequence of provided 


\begin{tabular}{lcc}
\hline \# tokens & out-of-domain & in-domain \\
\hline mean & $23.0 \pm 14.1$ & $6.6 \pm 4.8$ \\
median & 25 & 8 \\
max & 150 & 25 \\
\hline \hline \# lines & $5.5 \mathrm{M}$ & $1.3 \mathrm{M}$ \\
\hline
\end{tabular}

Table 2: Data statistics for source side of indomain and out-of-domain parallel data.

source sentences was the same for all participants, with no data intersection between the services beyond natural duplicates: About $11 \%$ of data were duplicates on both (DE and EN) sides, where about $4 \%$ of DE sentences had more than one different EN side.

Feedback. Simulation of real-valued user feedback was done by calculating the smoothed sentence-level BLEU-score (Lin and Och, 2004) (with additive $n$-gram count smoothing with offset 0.01 , applied only if the $n$-gram count was zero) with respect to one human reference (preprocessed as described above).

\section{Evaluation Metrics}

In our shared task, participants were allowed to use their favorite MT systems as starting points to integrate online bandit learning methods. This leads to the difficulty of separating the contributions of the underlying MT architecture and the online bandit learning method. We attempted to tackle this problem by using different evaluation metrics that focus on these respective aspects:

1. Online cumulative reward: This metric measures the cumulative sum $C=$ $\sum_{k=1}^{K} \Delta\left(t_{k}\right)$ of the per-sentence BLEU score $\Delta$ against the number of iterations. This metric has been used in reinforcement learning competitions (Dimitrakakis et al., 2014). For systems with the same design, this metric favors those that do a good job at balancing exploration and exploitation to achieve high scores over the full data sequence. Unlike in these competitions, where environments (i.e., action spaces and context features) were fixed, in our task the environment is heterogeneous due to the use of different underlying MT architectures. Thus, systems that start out with a well-performing pretrained out-of-domain model have an advantage over systems that might improve more over worse starting points. Furthermore, even systems that do not perform online learning at all can achieve high cumulative rewards.

2. Online regret: In order to overcome the problems of the cumulative reward metric, we can use a metric from bandit learning that measures the regret $R=\frac{1}{K} \sum_{k=1}^{K}\left(\Delta\left(t_{k}^{*}\right)-\right.$ $\left.\Delta\left(t_{k}\right)\right)$ that is suffered by the system when predicting translation $t_{k}$ instead of the optimal translation $t_{k}^{*}$ produced by an oracle system. Plotting a running average of regret against the number of iterations allows separating the gains due to the MT architecture from the gains due to the learning algorithm: Systems that do learn will decrease regret, systems that do not learn will not. In our task, we use as oracle system a model that is trained on in-domain data.

3. Relative reward: A further way to separate out the learning ability of systems from the contribution of the underlying MT architecture is to apply the standard corpus-BLEU score and/or an average of the per-sentence BLEU score $\Delta$ on a held-out set at regular intervals during training. Plotting these scores against the number of iterations, or alternatively, subtracting the performance of the starting point at each evaluation, allows to discern systems that adapt to a new domain from systems that are good from the beginning and can achieve high cumulative rewards without learning. We performed this evaluation by embedding a small (relative to the whole sequence) fixed held-out set in the beginning (showing the performance of the initial out-of-domain model), and again at regular intervals including the very end of the learning sequence. In total, there were 4 insertions of 700 sentences in the development data and 12 insertions of 4,000 sentences in the final training phase, which constitutes $\simeq 2 \%$ and $\simeq 0.3 \%$ of the respective learning sequence lengths. Note that this metric measures the systems' performance while they were still exploring and learning, but the relative size of the embedded held-out set is small enough to consider the models static during such periodic evaluations. 


\section{Baselines}

As baseline systems, we used SMT and NMT models that were trained on out-of-domain data, but did not perform online learning on in-domain data. We further present oracle systems that were trained in batch on in-domain data.

\subsection{Static SMT baselines.}

SMT-static. We based our SMT submissions on the SCFG decoder cdec (Dyer et al., 2010) with on-the-fly grammar extraction with suffix arrays (Lopez, 2007). Training was done in batch on the parallel out-of-domain data; tuning was done on newstest2016-deen. During the development phase we evaluated MERT (on 14 default dense features) and MIRA (on additional lexicalized sparse features: rule-id features, rule source and target bigram features, and rule shape features), and found no significant difference in results. We chose MERT with dense features as the seed system for the training phase for its speed and smaller memory footprint.

\subsection{Static NMT baselines.}

WMT16-static. First of all, we are interested in how well the currently best (third-party) model on the news domain would perform on the ecommerce domain. Therefore, the Nematus (Sennrich et al., 2017) model that won the News Translation Shared Task at WMT 2016 (Bojar et al., 2016b $)^{5}$ was used to translate the data from this shared task. It is an attentional, bidirectional, singe-layered encoder-decoder model on sub-word units (BPE with 89,500 merge operations) with word embeddings of dimensionality 500 , GRUs of size 1024, pervasive dropout and r21 reranking (details in (Sennrich et al., 2016a)). Final predictions are made with an ensemble formed of the four last training checkpoints and beam search with width 12 . It was trained on a different corpus than allowed for this shared task the WMT 2016 news training data (Europarl v7, News Commentary v11, CommonCrawl) and additional synthetic parallel data generated by translating the monolingual news crawl corpus with a EN-DE NMT model.

BNMT-static. The UNK replacement strategy of Jean et al. (2015) and Luong et al. (2015) is

\footnotetext{
${ }^{5}$ From data.statmt.org/rsennrich/wmt16 systems/de-en/
}

expected to work reasonable well for tokens that occur in the training data and those that are copied from source to target. However, the NMT model does not learn anything about these words as such in contrast to BPE models (Sennrich et al., 2016b) where the decomposition by byte pair encoding (BPE) allows for a representation within the vocabulary. We generate a BNMT system using a BPE vocabulary from 30k merge operations on all tokens and all single characters of the training data, including the UNK token. If unknown characters occur, they are copied from source to target.

\subsection{Oracle SMT and NMT systems}

To simulate full-information systems (oracles) for regret calculation, we trained an SMT and an NMT system with the same architectures, on the in-domain data that other learning systems accessed only through the numerical feedback. The SMT oracle system was trained on combined indomain and out-of-domain data, while the NMT oracle system continued training from the converged out-of-domain system on the in-domain data with the same BPE vocabulary.

\section{Submitted Systems}

\subsection{Online bandit learners based on SMT.}

Online bandit learners based on SMT were following the existing approaches to adapting an SMT model from weak user feedback (Sokolov et al., 2016b,a) by stochastically optimizing expected loss (EL) for a log-linear model. Furthermore, we present a model that implements stochastic zeroth-order (SZO) optimization for online bandit learning. Cube pruning limit (up to 600), learning rate adaptation schedules (constant vs. Adadelta (Zeiler, 2012) or Adam (Kingma and $\mathrm{Ba}, 2014)$ ), as well as the initial learning rates (for Adam), were tuned during the development phase. The best configurations were selected for the training phase. The running average of rewards as an additive control variate $(\mathrm{CV})^{6}$ was found helpful for stochastic policy gradient updates (Williams, 1992) for all online learning systems.

SMT-EL-CV-ADADELTA. We used the EL minimization approach of Sokolov et al. (2016a), adding Adadelta's learning rate scheduling, and a control variate (effectively, replacing the received

\footnotetext{
${ }^{6}$ Called a baseline in RL literature; here we use a term from statistics not to confuse it with baseline MT models.
} 
feedback $\Delta\left(t_{k}\right)$ with $\left.\Delta\left(t_{k}\right)-\frac{1}{k} \sum_{k^{\prime}=1}^{k} \Delta\left(t_{k^{\prime}}\right)\right)$. Sampling and computation of expectations on the hypergraph used the Inside-Outside algorithm $(\mathrm{Li}$ and Eisner, 2009).

SMT-EL-CV-ADAM. This system uses the same approach as above except for using Adam to adapt the learning rates, with tuning of the initial learning rate on the development service.

SMT-SZO-CV-ADAM. As a novel contribution, we adapted the two-point stochastic zerothorder approach by (Sokolov et al., 2015) that required two quality evaluation per iteration to a one-point feedback scenario. In a nutshell, on each step of the SZO algorithm, the model parameters $w$ are perturbed with an additive standard Gaussian noise $\epsilon$, and the Viterbi translation is sent to the service. Such algorithm can be shown to maximize the smoothed version of the task reward: $\mathbb{E}_{\epsilon \sim N(0,1)}[\Delta(\hat{y}(w+\epsilon))]$ (Flaxman et al., 2005). The advantages of such a black-box optimization method over model-based (e.g. EL) optimization, that requires sampling of complete structures from the model distribution, are simpler sampling of standard Gaussians, and matching of the inference criterion to the learning objective (MAP inference for both), unlike the EL optimization of expected reward that is still evaluated at test time using MAP inference. For SZO models we found that the Adam scheduling consistently outperforms Adadelta.

\subsection{Online bandit learners based on NMT.}

Kreutzer et al. (2017) recently presented an algorithm for online expected loss minimization to adapt NMT models to unknown domains with bandit feedback. Exploration (i.e. sampling from the model) and exploitation (i.e. presenting the highest scored translation) are controlled by the softmax distribution in the last layer of the network. Ideally, the model would converge towards a peaked distribution. In our online learning scenario this is not guaranteed, but we would like the model to gradually stop exploring, in order to still achieve high cumulative per-sentence reward. To achieve such a behavior, the temperature of the softmax over the outputs of the last layer of the network is annealed (Rose, 1998). More specifically, let $o$ be the scores of the output projection layer of the decoder, then $p_{\theta}\left(\tilde{y}_{t}=w_{i} \mid \mathbf{x}, \hat{\mathbf{y}}_{<t}\right)=$ $\frac{\exp \left(o_{w_{i}} / T\right)}{\sum_{v=1}^{V} \exp \left(o_{w_{v}} / T\right)}$ is the distribution that defines the probability of each word $w_{i}$ of the target vocabulary $V$ to be sampled in timestep $t$. The annealing schedule for this temperature $T$ is defined as $T_{k}=0.99^{\max \left(k-k_{\mathrm{START}}, 0\right)}$, i.e. decreases from iteration $k_{\text {START }}$ on. The same decay is applied to the learning rate, such that $\gamma_{k}=\gamma_{k-1} \cdot T_{k}$. This schedule was proven successful during tuning with the leaderboard.

WNMT-EL. Using the implementation of Kreutzer et al. (2017), we built a word-based NMT system with NeuralMonkey (Libovickỳ et al., 2016; Bojar et al., 2016a) and trained it with the EL algorithm. The vocabulary is limited to the $30 \mathrm{k}$ most frequent words in the out-of-domain training corpus. The architecture is similar to WMT16-static with GRU size 1024, embedding size 500. It was pretrained on the out-of-domain data with the standard maximum likelihood objective, Adam $\left(\alpha=1 \times 10^{-4}\right.$, $\left.\beta_{1}=0.9, \beta_{2}=0.999\right)$ and dropout (Srivastava et al., 2014) with probability 0.2. Bandit learning starts from this pretrained model and continues with stochastic gradient descent (initial learning rate $\gamma_{0}=1 \times 10^{-5}$, annealing starts at $k_{S T A R T}=700,000$, dropout with probability 0.5 , gradient norm clipping when the norm exceeds 1.0 (Pascanu et al., 2013)), where the model was updated as soon as a feedback is received. As described above, UNK replacement was applied to the output on the basis of an IBM2 lexical translation model built with fast_align (Dyer et al., 2013) on out-of-domain training data. If the aligned source word for a generated UNK token is not in the dictionary of the lexical translation model, the UNK token was simply replaced by the source word.

BNMT-EL. The pretrained BPE model is further trained on the bandit task data with the EL algorithm, as described for BL1, with the only difference of using Adam $\left(\alpha=1 \times 10^{-5}, \beta_{1}=0.9\right.$, $\left.\beta_{2}=0.999\right)$ instead of SGD. Again, annealing started at $k_{S T A R T}=700,000$.

BNMT-EL-CV. BNMT-EL-CV is trained in the same manner as BNMT-EL with the addition of the same control variate technique (running average of rewards) that has been previously found to improve both variance and generalization for NMT bandit training (Kreutzer et al., 2017). 


\subsection{Domain adaptation and reinforcement learning based on NMT (University of Maryland).}

UMD-domain-adaptation. The UMD team's systems were based on an attention-based encoder-decoder translation model. The models use the BPE technique for subword encoding, which helps addressing the rare word problem and enlarges vocabulary. A further addition is the domain adaptation approach of Axelrod et al. (2011) to select training data after receiving in-domain source-side data and selecting the most similar out-of-domain data from the WMT 2016 training set for re-training.

UMD-reinforce. Another type of models submitted by UMD uses reinforcement learning techniques to learn from feedback and improve the update of the translation model to optimize the reward, based on Bahdanau et al. (2016) and Ranzato et al. (2016).

\subsection{Domain adaptation and bandit learning based on SMT (LIMSI).}

LIMSI. The team from LIMSI tried to adapt a seed Moses system trained on out-domain data to a new, unknown domain relying on two components, each of which addresses one of the challenges raised by the shared task: i) estimate the parameters of a MT system without knowing the reference translation and in a 'one-shot' way (each source sentence can only be translated once); ii) discover the specificities of the target domain 'onthe-fly' as no information about it is available. First, a linear regression model was used to exploit weak and partial feedback the system received by learning to predict the reward a translation hypothesis will get. This model can then be used to score hypotheses of the search space and translate source sentences while taking into account the specificities of the in-domain data. Second, three variants of the UCB1 (Auer et al., 2002) algorithm (vanilla UCB1, a UCB1-sampling variant encouraging more exploration, and a UCB1 with selecting only the examples not used to train the regression model) chose which of the 'adapted' or 'seed' systems should be used to translate a given source sentence in order to maximize the cumulative reward (Wisniewski, 2017).

\begin{tabular}{|c|c|c|}
\hline & model & $\begin{array}{c}\text { cumulative } \\
\text { reward }\end{array}$ \\
\hline & 'translate' by copying source & $64,481.8$ \\
\hline \multirow{2}{*}{$\sum_{n}^{E}$} & $\begin{array}{l}\text { SMT-oracle } \\
\text { SMT-static }\end{array}$ & $\begin{array}{l}499,578.0 \\
229,621.7\end{array}$ \\
\hline & $\begin{array}{l}\text { SMT-EL-CV-ADADELTA } \\
\text { SMT-EL-CV-ADAM } \\
\text { SMT-SZO-CV-ADAM }\end{array}$ & $\begin{array}{l}214,398.8 \\
225,535.3 \\
208,464.7\end{array}$ \\
\hline \multirow{3}{*}{$\sum_{Z}^{E}$} & $\begin{array}{l}\text { BNMT-oracle } \\
\text { BNMT-static } \\
\text { WMT16-static }\end{array}$ & $\begin{array}{l}780,580.4 \\
222,066.0 \\
139,668.1\end{array}$ \\
\hline & $\begin{array}{l}\text { BNMT-EL-CV } \\
\text { BNMT-EL } \\
\text { WNMT-EL }\end{array}$ & $\begin{array}{l}212,703.2 \\
237,663.0 \\
115,098.0\end{array}$ \\
\hline & UMD-domain-adaptation & $248,333.2$ \\
\hline
\end{tabular}

Table 3: Cumulative rewards over the full training sequence. Only completely finished submission are shown.

\section{Results}

Table 3 shows the evaluation results under the cumulative rewards metric. Of the non-oracle systems, good results are obtained by static SMT and BNMT system, while the best performance is obtained by the UMD-domain adaptation system which is also basically a static system. This result is followed closely by the online bandit learner BNMT-EL which is based on an NMT baseline and optimizes the EL objective. It outperforms the BNMT-static baseline. Cumulative rewards could not be computed for all submitted systems since some training runs could not be fully finished.

The evolution of the online regret plotted against the log-scaled number of iterations during training is shown in Figure 1. Most of the learning happens in the first 100,000 iterations, however, online learning systems optimizing structured EL objectives or based on reinforcement learning eventually converge to the same result: BNMT-EL or UMD-reinforce2 get close to the regret of the static UMD-domain adaptation. Systems that optimize the EL objective do not start from strong out-of-domain systems with domainadaptation, however, due to a steeper learning curve they arrive at similar results.

Figures 2, 3a and $3 \mathrm{~b}$ show the evolution of corpus- and sentence-BLEU on the heldout set that 


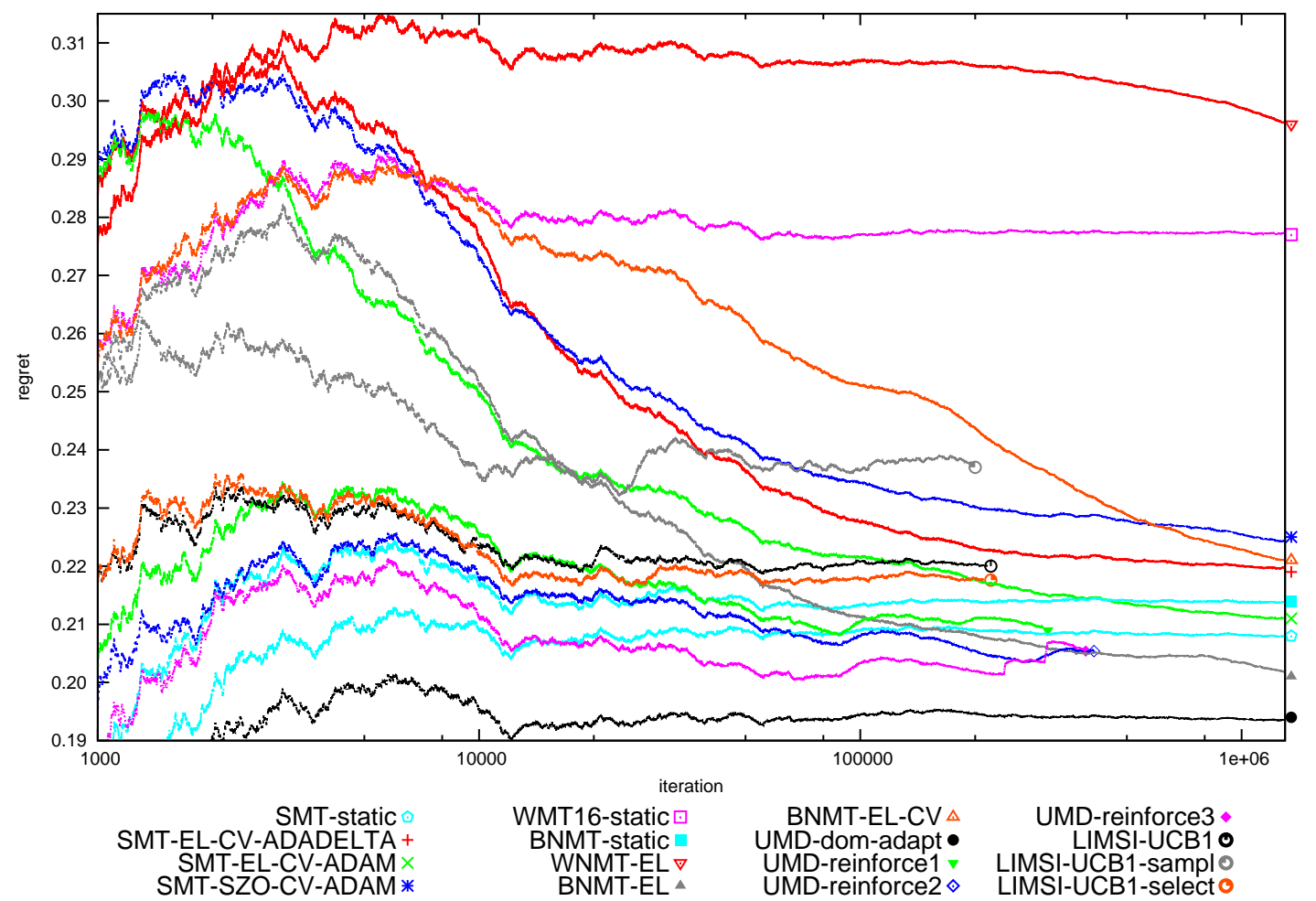

Figure 1: Evolution of regret plotted against log-scaled number of iterations during training. The steeper is the decrease of a curve, the better learning capability has the corresponding algorithm.

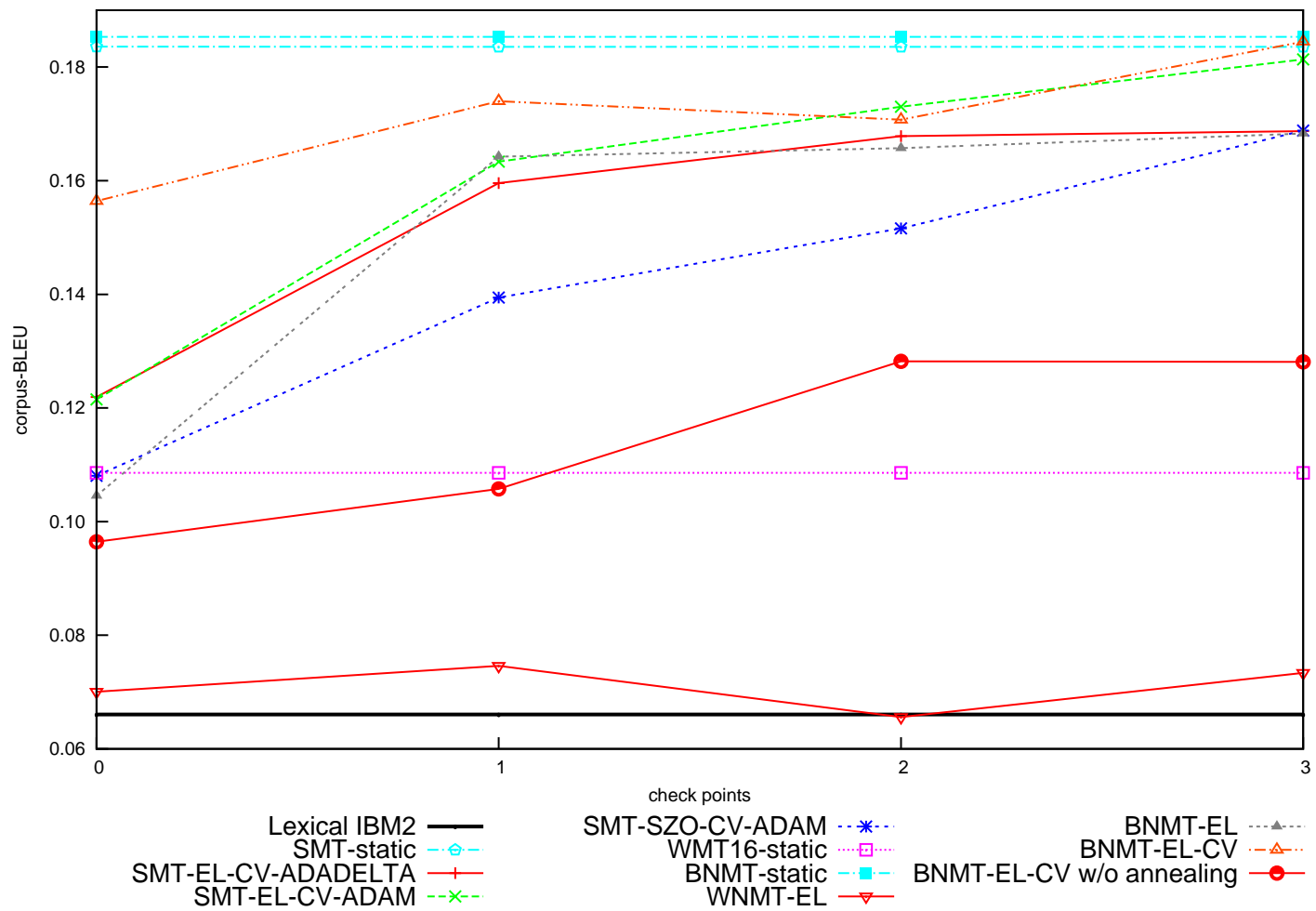

Figure 2: Evolution of corpus BLEU scores during development for configuration selected for the training phase of the competition. Each check point is comprised of the same 700 sentences spaced at a regular intervals of 12,400 sentences starting from the beginning of the development sequence. 

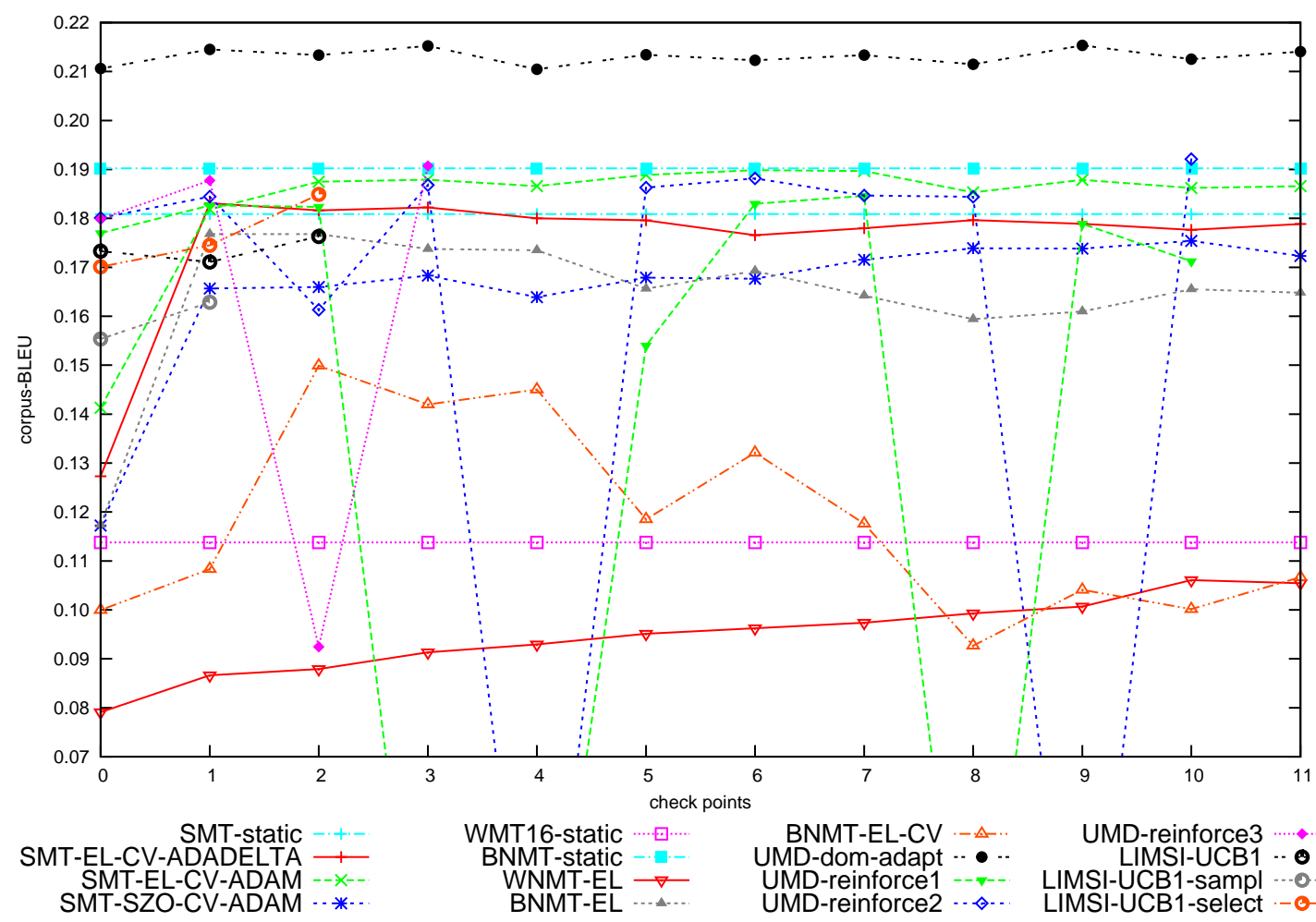

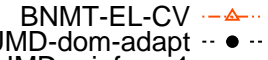
UMD-reinforce 1 UMD-reinforce2

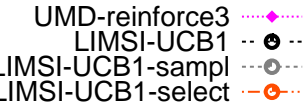

(a) corpus-BLEU

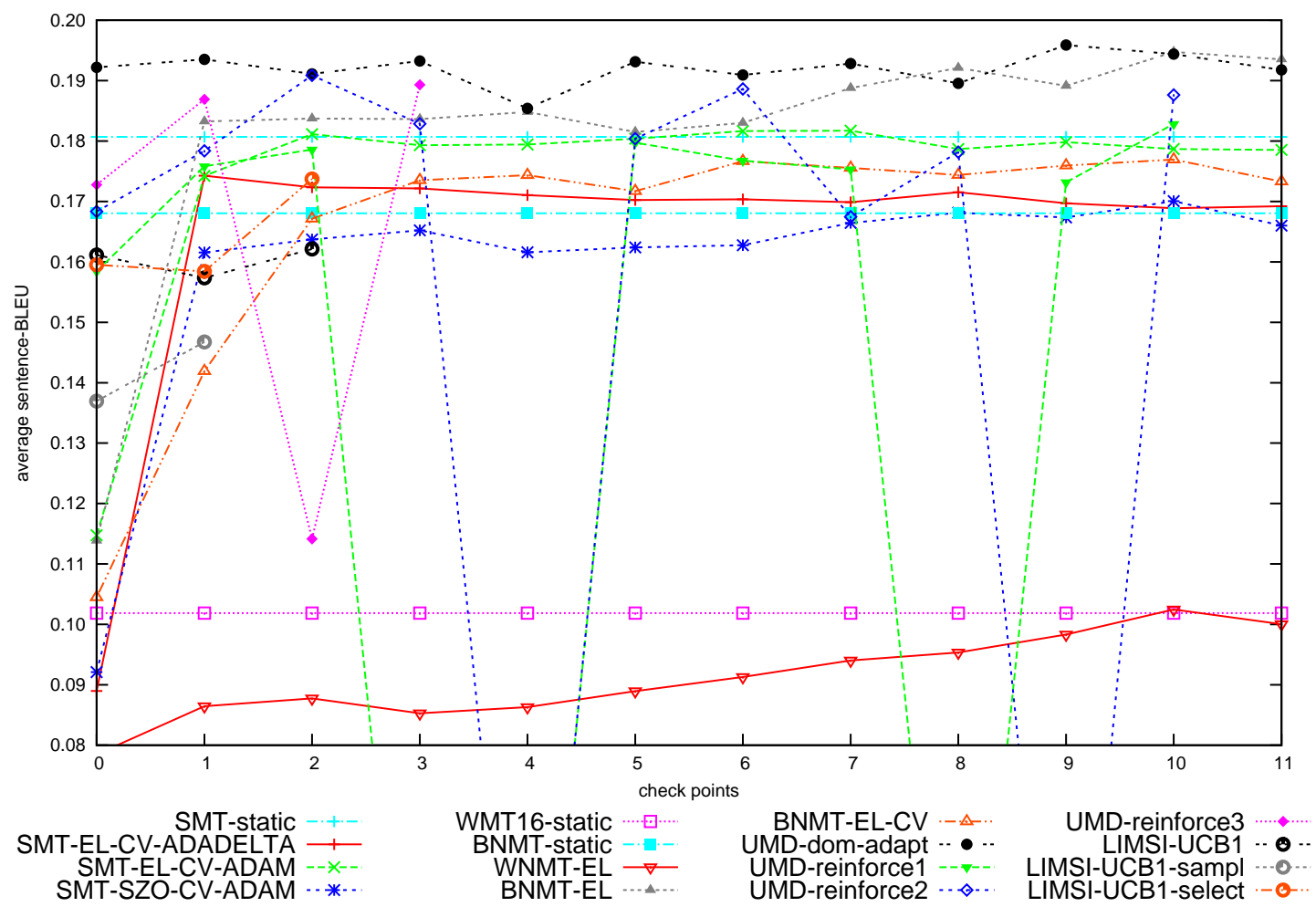

(b) sentence-BLEU

Figure 3: The evolution of corpus- and sentence-BLEU scores during training for all participant and baselines. Each check point is comprised of the same 4,000 sentences spaced at a regular intervals of 113,634 sentences starting from the beginning of the training sequence. 
has been embedded in the development and the training sequences. While under corpus-BLEU, static systems always outperform online learners on the held-out embedded set, online learning systems such as BNMT-EL can catch up under corpus-BLEU during development, and under a sentence-BLEU evaluation during training. The curves for corpus- and average sentence-BLEU (Figures $3 \mathrm{a}$ and $3 \mathrm{~b}$ ) show a different dynamics, with the corpus-BLEU sometimes decreasing whereas the sentence-BLEU curve continues to increase. However, if the focus is online learning, the online task loss is per-sentence BLEU and so should be the evaluation metric.

\section{Conclusion}

We presented the learning setup and infrastructure, data and evaluation metrics, and descriptions of baselines and submitted systems for a novel shared task on bandit learning for machine translation. The task implicitly involved domain adaptation from the news domain to e-commerce data (with the additional difficulty of non-literal post-editions as references), and online learning from simulated per-sentence feedback on translation quality (creating a mismatch between the persentence task loss and the corpus-based evaluation metric standardly used in evaluating batch-trained machine translation systems). Despite these challenges, we found promising results for both linear and non-linear online learners that could outperform their static SMT and NMT baselines, respectively. A desideratum for a future installment of this shared task is the option to perform offline learning from bandit feedback (Lawrence et al., 2017), thus allowing a more lightweight infrastructure, and opening the task to (mini)batch learning techniques that are more standard in the field of machine translation.

\section{Acknowledgments}

This research was supported in part by the German research foundation (DFG), and in part by a research cooperation grant with the Amazon Development Center Germany. We would like to thank Amazon for supplying data and engineering expertise, and for covering the running costs.

\section{References}

Peter Auer, Nicolò Cesa-Bianchi, and Paul Fischer. 2002. Finite-time analysis of the multiarmed bandit problem. Machine Learning 47(2-3):235-256.

Amittai Axelrod, Xiaodong He, and Jianfeng Gao. 2011. Domain adaptation via pseudo in-domain data selection. In EMNLP. Edinburgh, Scotland.

Dzmitry Bahdanau, Philemon Brakel, Kelvin Xu, Anirudh Goyal, Ryan Lowe, Joelle Pineau, Aaron Courville, and Yoshua Bengio. 2016. An ActorCritic Algorithm for Sequence Prediction. eprint arXiv:1607.07086

Ondřej Bojar, Roman Sudarikov, Tom Kocmi, Jindřich Helcl, and Ondřej Cifka. 2016a. UFAL submissions to the IWSLT 2016 MT track. In IWSLT. Seattle, WA.

Ondřej Bojar, Rajen Chatterjee, Christian Federmann, Yvette Graham, Barry Haddow, Matthias Huck, Antonio Jimeno Yepes, Philipp Koehn, Varvara Logacheva, Christof Monz, Matteo Negri, Aurelie Neveol, Mariana Neves, Martin Popel, Matt Post, Raphael Rubino, Carolina Scarton, Lucia Specia, Marco Turchi, Karin Verspoor, and Marcos Zampieri. 2016b. Findings of the 2016 conference on machine translation. In WMT. Berlin, Germany.

Sébastian Bubeck and Nicolò Cesa-Bianchi. 2012. Regret analysis of stochastic and nonstochastic multiarmed bandit problems. Foundations and Trends in Machine Learning 5(1):1-122.

Olivier Chapelle, Eren Masnavoglu, and Romer Rosales. 2014. Simple and scalable response prediction for display advertising. ACM Trans. on Intelligent Systems and Technology 5(4).

Christos Dimitrakakis, Guangliang Li, and Nikolaos Tziortziotis. 2014. The reinforcement learning competition 2014. AI Magazine 35(3):61-65.

Chris Dyer, Victor Chahuneau, and Noah A. Smith. 2013. A simple, fast, and effective reparameterization of IBM model 2. In HLT-NAACL. Atlanta, GE.

Chris Dyer, Adam Lopez, Juri Ganitkevitch, Jonathan Weese, Ferhan Ture, Phil Blunsom, Hendra Setiawan, Vladimir Eidelman, and Philip Resnik. 2010. cdec: A decoder, alignment, and learning framework for finite-state and context-free translation models. In ACL Demo. Uppsala, Sweden.

Abraham D. Flaxman, Adam Tauman Kalai, and H. Brendan McMahan. 2005. Online convex optimization in the bandit setting: gradient descent without a gradient. In SODA. Vancouver, Canada.

Yvette Graham, Timothy Baldwin, Alistair Moffat, and Justin Zobel. 2016. Can machine translation systems be evaluated by the crowd alone? Natural Language Engineering 23(1):3-30. 
Sébastien Jean, Orhan Firat, Kyunghyun Cho, Roland Memisevic, and Yoshua Bengio. 2015. Montreal neural machine translation systems for WMT'15. In WMT. Lisbon, Portugal.

Diederik Kingma and Jimmy Ba. 2014. Adam: A method for stochastic optimization. eprint arXiv:1412.6980 .

Julia Kreutzer, Artem Sokolov, and Stefan Riezler. 2017. Bandit structured prediction for neural sequence-to-sequence learning. In $A C L$. Vancouver, Canada.

Carolin Lawrence, Artem Sokolov, and Stefan Riezler. 2017. Counterfactual learning from bandit feedback under deterministic logging: A case study in statistical machine translation. In EMNLP. Copenhagen, Denmark.

Zhifei Li and Jason Eisner. 2009. First-and secondorder expectation semirings with applications to minimum-risk training on translation forests. In EMNLP. Singapore.

Jindřich Libovickỳ, Jindřich Helcl, Marek Tlustỳ, Pavel Pecina, and Ondřej Bojar. 2016. CUNI system for WMT16 automatic post-editing and multimodal translation tasks. In WMT. Berlin, Germany.

Chin-Yew Lin and Franz Josef Och. 2004. Automatic evaluation of machine translation quality using longest common subsequence and skip-bigram statistics. In ACL. Barcelona, Spain.

Adam Lopez. 2007. Hierarchical phrase-based translation with suffix arrays. In EMNLP-CoNLL. Prague, Czech Republic.

Thang Luong, Ilya Sutskever, Quoc Le, Oriol Vinyals, and Wojciech Zaremba. 2015. Addressing the rare word problem in neural machine translation. In $A C L$. Beijing, China.

Razvan Pascanu, Tomas Mikolov, and Yoshua Bengio. 2013. On the difficulty of training recurrent neural networks. In ICML. Atlanta, GA.

MarcAurelio Ranzato, Sumit Chopra, Michael Auli, and Wojciech Zaremba. 2016. Sequence level training with recurrent neural networks. In ICLR. San Juan, Puerto Rico.

Kenneth Rose. 1998. Deterministic annealing for clustering, compression, classification, regression and related optimization problems. IEEE 86(11).

Rico Sennrich, Orhan Firat, Kyunghyun Cho, Alexandra Birch, Barry Haddow, Julian Hitschler, Marcin Junczys-Dowmunt, Samuel Läubli, Antonio Valerio Miceli Barone, Jozef Mokry, and Maria Nadejde. 2017. Nematus: a toolkit for neural machine translation. In EACL. Valencia, Spain.

Rico Sennrich, Barry Haddow, and Alexandra Birch. 2016a. Edinburgh neural machine translation systems for WMT 16. In WMT. Berlin, Germany.
Rico Sennrich, Barry Haddow, and Alexandra Birch. 2016b. Neural machine translation of rare words with subword units. In ACL. Berlin, Germany.

Artem Sokolov, Julia Kreutzer, Christopher Lo, and Stefan Riezler. 2016a. Learning structured predictors from bandit feedback for interactive NLP. In $A C L$. Berlin, Germany.

Artem Sokolov, Julia Kreutzer, Christopher Lo, and Stefan Riezler. 2016b. Stochastic structured prediction under bandit feedback. In NIPS. Barcelona, Spain.

Artem Sokolov, Stefan Riezler, and Tanguy Urvoy. 2015. Bandit structured prediction for learning from partial feedback in statistical machine translation. In MT Summit. Miami, FL.

Nitish Srivastava, Geoffrey E Hinton, Alex Krizhevsky, Ilya Sutskever, and Ruslan Salakhutdinov. 2014. Dropout: a simple way to prevent neural networks from overfitting. JMLR 15(1):1929-1958.

Richard S. Sutton and Andrew G. Barto. 1998. Reinforcement Learning. An Introduction. The MIT Press.

Csaba Szepesvári. 2009. Algorithms for Reinforcement Learning. Morgan \& Claypool.

Ronald J. Williams. 1992. Simple statistical gradientfollowing algorithms for connectionist reinforcement learning. Machine Learning 20:229-256.

Guillaume Wisniewski. 2017. LIMSI submission for WMT'17 shared task on bandit learning. In WMT. Copenhagen, Denmark.

Matthew D. Zeiler. 2012. ADADELTA: an adaptive learning rate method. eprint arXiv:1212.5701 . 